\title{
International Cancer Genome Consortium
}

Etwa zehn Jahre hatte es gedauert, das Genom des Menschen zu entschlüsseln. Einen nächsten Schritt bildete das 1.000-Genom-Projekt. Ziel war es, jede Genvariante zu finden, die bei mindestens einem

a Prozent der Menschen vorkommt; nicht zuletzt, um mögliche Zusammenhänge zwischen einem bestimmten Gen und einer Krankheit aufzudecken. Im Jahre 2008 schlossen sich weltweit Krebsforscher zum „International Cancer Genome Consortium" (ICGC) zusammen. Seit 2010 sind auch deutsche Wissenschaftler daran beteiligt, Treibermutationen der 50 wichtigsten Tumoren zu identifizieren. Das Bundesministerium für Bildung und Forschung und die Deutsche Krebshilfe e. V. fördern die deutsche ICGC-Beteiligung über fünf Jahre mit 15 Millionen Euro.

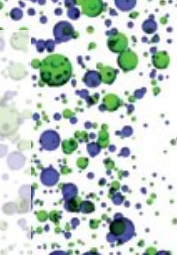

Co ?ain 8 \begin{tabular}{l}
0 \\
$\because$ \\
\hdashline \\
0
\end{tabular}

\section{-}

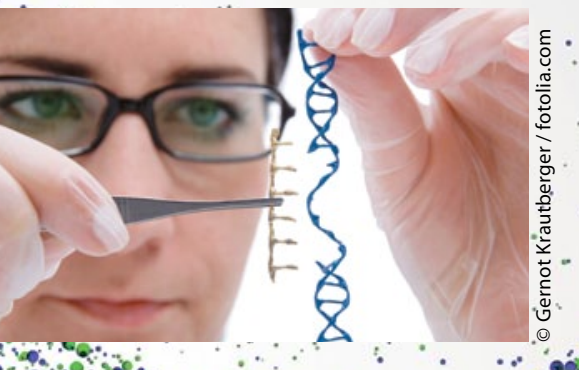

8 a o.

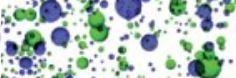
as âçhes dieser Forschungsgebiete $\therefore \quad$ liegt in deutscher Verantwortung?

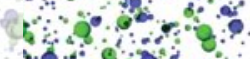
000

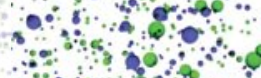

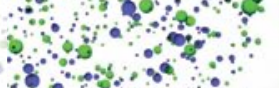
800 $\because \because 3$

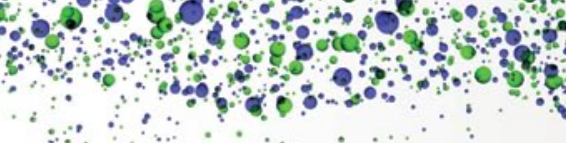

A Zervixkarzinom Plattenepithelkarżinom der Zervix

B Pankreaskarzinom duktales Adenokarzinom

C Malignes Lymphom Keimzentrums-B-Zell-Lymphom
Der Buchstabe der richtigen Antwort ist

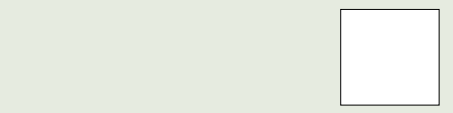

Name

Straße, Nr.

PLZ, Ort

Was ich noch sagen wollte...

Coupon bitte ausfüllen und abschicken an Springer Medizin, Urban \& Vogel GmbH

Redaktion Im Focus Onkologie - Quiz 5/2013

Aschauer Straße 30 • 81549 München

Oder senden Sie uns eine E-Mail an doris.berger@springer.com

Einsendeschluss: 18.06.2013
Q Oilo das können sie gewinnen ... \$. $94: 80 \%$

was Wissen schafft.

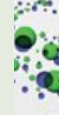

the

Gutscheila über $50 €$

me

ât8 000

Q. Springer Medizin

Unter den richtigen

Einsendungen verlosen

wir drei Gutscheine über

50 Euro, die Sie für ein

beliebiges Springer-Buch

einlösen können.

Einsendeschluss ist der

18.06.2013.
Lösung des Quiz 3/2013

Richtig war: A

Die Gewinne gehen an:

N. Otto, 63843 Niedernberg

Ch. Pilz, 91077 Neunkirchen

M. Kirrbach, 09217 Burgstädt

Herzlichen Glückwunsch! 